\title{
Ocular Tissue
}

National Cancer Institute

\section{Source}

National Cancer Institute. Ocular Tissue. NCI Thesaurus. Code C133340.

Different types of tissues from the human eye. 